\title{
Probabilistic Concepts in a Changing Climate: A Snapshot Attractor Picture*
}

\author{
GÁBOR DRÓTOS \\ Institute for Theoretical Physics, Eötvös Loránd University, and MTA-ELTE Theoretical Physics Research Group, \\ Budapest, Hungary \\ TAMÁS BÓDAI \\ Meteorological Institute, University of Hamburg, Hamburg, Germany \\ TAMÁS TÉL \\ Institute for Theoretical Physics, Eötvös Loránd University, and MTA-ELTE Theoretical Physics Research Group, \\ Budapest, Hungary
}

(Manuscript received 1 July 2014, in final form 29 October 2014)

\begin{abstract}
The authors argue that the concept of snapshot attractors and of their natural probability distributions are the only available tools by means of which mathematically sound statements can be made about averages, variances, etc., for a given time instant in a changing climate. A basic advantage of the snapshot approach, which relies on the use of an ensemble, is that the natural distribution and thus any statistics based on it are independent of the particular ensemble used, provided it is initiated in the past earlier than a convergence time. To illustrate these concepts, a tutorial presentation is given within the framework of a low-order model in which the temperature contrast parameter over a hemisphere decreases linearly in time. Furthermore, the averages and variances obtained from the snapshot attractor approach are demonstrated to strongly differ from the traditional 30-yr temporal averages and variances taken along single realizations. The authors also claim that internal variability can be quantified by the natural distribution since it characterizes the chaotic motion represented by the snapshot attractor. This experience suggests that snapshot-attractor-based calculations might be appropriate to be evaluated in any large-scale climate model, and that the application of 30-yr temporal averages taken along single realizations should be complemented with this more appealing tool for the characterization of climate changes, which seems to be practically feasible with moderate ensemble sizes.
\end{abstract}

\section{Introduction}

It is common to talk about climate changes in statistical terms, in a naive sense at least. Computer climate models are at the heart of climate science, as a tool of producing analysis, but also as a subject of development. Such models are used to simulate past climate changes and future climate change scenarios. These changes come

\footnotetext{
* Supplemental information related to this paper is available at the Journals Online website: http://dx.doi.org/10.1175/JCLI-D-1400459.s1.

Corresponding author address: Gábor Drótos, Institute for Theoretical Physics, Eötvös University, Pázmány Péter sétány 1/A, H-1117 Budapest, Hungary.

E-mail: drotos@general.elte.hu
}

about due to a shift in some model parameter (e.g., the concentration of atmospheric $\left.\mathrm{CO}_{2}\right)$. Ensemble simulations are widely used nowadays (see e.g., Stocker et al. 2013; Taylor et al. 2012), including calculations where the results depend on the choice of initial data (Deser et al. 2012a,b). Unlike what is done in most of the ensemble simulations and is claimed in Stocker et al. (2013, chapter 12 ), in our paper we would like to argue that it is worth separating internal variability from other sources of uncertainties (Hawkins and Sutton 2009; Stocker et al. 2013), since this source has a dynamical origin (while the others have an experimental or a societal origin). As a consequence of this origin, internal variability turns out to be describable by a well-defined probability distribution. This probability distribution is obtained from an ensemble of trajectories (that differ solely in their initial conditions and not, e.g., in their parameters), 
rather than from the time evolution of a particular single realization. In a wider sense, this kind of framework has been suggested as a novel way of treating the climate system (Hasselmann 1976; Paillard 2008; R. T. Pierrehumbert 2011, personal communication; Bódai and Tél 2012; Ghil 2012; S. Stainforth 2012, personal communication) by claiming that the relevant quantities are the statistics taken over an ensemble of possible realizations. In our paper we illustrate the relevant probability distribution to be unique (i.e., independent of the particular set of initial conditions of the ensemble). To see the unique distribution one has to consider any specific ensemble after a finite convergence time has passed from the initialization. This also means that in our paper uninitialized climate projections are concerned in the terminology of Stocker et al. (2013).

The mathematical concept that fits perfectly to our picture is that of snapshot attractors (we shall use this term throughout the paper) (Romeiras et al. 1990) or pullback attractors (Arnold 1998; Ghil et al. 2008), arising in the study of dissipative nonautonomous systems (Arnold 1998; Kloeden and Rasmussen 2011; Carvalho et al. 2014). Loosely speaking, a snapshot attractor, as introduced by Romeiras et al. (1990), is an object belonging to a given time instant that is traced out by an ensemble of trajectories initialized in the remote past, while all of them are governed by the same equation of motion. A pullback attractor is a similar object associated with the entire real-time axis $(-\infty<t<\infty)$. A rigorous mathematical definition only exists for the pullback attractor, and according to this definition, given by Ghil et al. (2008) and Chekroun et al. (2011), an initialization of the ensemble in the infinite past is required. This property enables one to show the existence of singular measures on such attractors. The aforementioned mathematical definition requires also the consideration of the dynamics' two-time evolution operator (Sell 1967a,b; Gaspard 2005; Crauel and Flandoli 1994; Crauel et al. 1997; Young 2002; Ghil et al. 2008). As follows from all this, a snapshot attractor can be viewed as an instantaneous slice (corresponding to a given time instant) of a pullback attractor. In the dynamical systems community, the concept of snapshot attractors has been known for many years (Romeiras et al. 1990; Yu et al. 1990; Jacobs et al. 1997; Hansen and Bohr 1998; Neufeld and Tél 1998; Károlyi et al. 2004; Bódai et al. 2011b) and explains even experimental findings (Sommerer and Ott 1993).

An appealing feature of snapshot attractors is that they carry unique probability distributions that are independent of the particular ensemble used for their representation. They trace out clear structures, whereas a traditional single trajectory plot would lead in the same problem to an unstructured pattern. With chaotic dynamics, the snapshot attractor is a fractal, whose shape changes over time, but its fractal dimension might stay constant (Ledrappier and Young 1988; Romeiras et al. 1990). The existence of such an attractor is rather nontrivial in the sense that one might expect to have different sets of end points belonging to ensembles initiated in different time instants in the past (e.g., similar to the case of conservative systems), while this turns out to be untrue here.

Regarding the concept of climate, Ghil and coworkers (Ghil et al. 2008; Chekroun et al. 2011) were the first to call the attention to the relevance of snapshot (or pullback) attractors. They have been used so far (Bódai and Tél 2012; Pierini 2012; Bódai et al. 2013; Crucifix 2013; Ghil 2015) mainly to visualize stochastic effects as well as periodic external forcing. Ghil et al. (2008) and Chekroun et al. (2011) have also claimed them to be useful tools in climate change science, incorporating stochastic and/or deterministic forcing. In our paper we argue that the use of snapshot attractors is most natural, and, even more, seems to be unavoidable, when dealing with climate changes induced by smoothly shifting parameters, without including any stochasticity. A smooth shift is a special case of an aperiodic forcing. In this situation the snapshot framework is the only one in which a probability distribution, the well-defined instantaneous distribution (in mathematical terms, the natural measure) on the snapshot attractor, can be associated with the dynamics. This distribution is time dependent. We claim that any statistics associated with specific time instants should be taken with respect to this distribution. In addition, we claim that climate changes can also be seen as the change of snapshot attractors and their natural distributions [as also mentioned by Bódai and Tél (2012)].

The above approach is proposed to complement the traditional one, based on 30-yr temporal statistics along a single realization (i.e., a single time series of recorded or that of simulated data). The traditional definition appears in Stocker et al. (2013) [see also Holton (2004, chapter 10)], in which the considered statistics can be some low-order moment (e.g., average, variance, skewness) or a linear or nonlinear trend (Ghil and Vautard 1991; Ji et al. 2014; Franzke 2014) that a single realization of the time series constitutes. A conceptual difference between the new and the traditional approach is that the data derived from the traditional one depend on the particular realization and thus cannot reflect the behavior of all possible realizations. We show in this paper that in general dissipative nonautonomous problems the single realization and the snapshot pictures lead to very different results indeed. 
An implicit-although, in view of the previous paragraph, unrealistic-aim of the traditional approach might be the extraction of information from the time evolution of one single realization on the probabilities associated with the ensemble of all possible realizations. This is, however, impossible in general. In fact, there exists only one exceptional situation when this can be done: this is the case of constant or temporally periodic forcing. Even then only infinitely long time averages turn out to be equivalent with ensemble averages [the system is then ergodic in the sense of statistical physics; Reichl (1998)].

Within the snapshot attractor picture novel features of our work are the consideration of a deterministic and linearly changing forcing, the illustration of the finiteness of the convergence time toward the snapshot attractor, and the comparison of snapshot averages with 30-yr temporal averages taken along individual time series.

For these purposes, we turn to a very simple conceptual climate model. It is Lorenz's model of atmospheric circulation (Lorenz 1984, 1990) described by three ordinary differential equations. Such simple models play an important role in climate research (Provenzale and Balmforth 1999), as they expedite efficient testing of theoretical ideas and facilitate simple visualization of the results. They can give thus a proof of concept for novel approaches. As a modification to Lorenz's model, in harmony with the discussion above, we incorporate into the driving a monotonic (linear) shift, called the ramp, in the temperature contrast parameter. Without this shift, but including the seasonal cycle (Lorenz 1990), we say that the climate is stationary (i.e., not changing); with the inclusion of the ramp in addition, however, a changing climate is modeled.

The paper is organized as follows. In section 2 we shortly introduce the model outlined above. In section 3 we point out that the case of the stationary climate is characterized by a usual chaotic attractor, on which infinitely long time averages are equivalent to ensemble averages. This is, however, not the case with the ramp, when only the snapshot picture enables one to define a probability distribution characterizing a given time instant, as detailed in section 4 . We claim that this distribution represents the internal variability, and that the temporal change of its average provides an example for the forced response. Next, in section 5, single-realization statistics taken over some time interval are considered, and are shown to give quantitatively different results from those of the snapshot picture. We thus conclude that they cannot be used for obtaining useful hints on the relevant instantaneous probabilities. The last section contains our final remarks, with a discussion, among others, of an extension of the snapshot picture to ensemble averages of 30-yr temporal averages. The evaluation of different snapshot-type statistics might thus be appropriate in any large-scale climate model, in which direction the first steps were made by Deser et al. (2012a,b) when estimating the internal variability from ensemble runs, although without assuring the convergence of their ensemble of initial conditions to a dynamical attractor. To not break the tutorial form of the presentation, issues of technical character are relegated to the appendix and to the supplemental material.

\section{The model}

The physical content of Lorenz's atmospheric circulation model for the midlatitudes on one hemisphere is the following. Solar forcing creates a temperature difference between the equator and the pole, which is proportional to the model variable $F$, and it influences most directly the wind speed of the westerlies represented by $x$. As an effect of baroclinic instability, cyclonic activity facilitates poleward heat transport, two modes of which are represented by $y$ and $z$. This appealing low-order model was studied in different contexts (Masoller and Schifino 1992; Pielke and Zeng 1994; Masoller et al. 1995; Nicolis et al. 1995; Roebber 1995; Shil'nikov et al. 1995; Provenzale and Balmforth 1999; Leonardo 1995; Tél and Gruiz 2006; Freire et al. 2008; Bódai et al. 2011a, 2013). The model reads as follows:

$$
\begin{aligned}
& \dot{x}=-y^{2}-z^{2}-a x+a F(t), \\
& \dot{y}=x y-b x z-y+G, \\
& \dot{z}=x z+b x y-z .
\end{aligned}
$$

For the parameter setting we take the common choice: $a=1 / 4, b=4$, and $G=1$ (Lorenz 1984). The equations appear in a dimensionless form with the time unit corresponding to 5 days, according to Lorenz (1984). The constant value of $F(t)=F_{0}=6$ (8) was regarded by Lorenz to be an appropriate value for permanent summer (winter), and the system with this value exhibits only periodic (chaotic) attractors. We note that the model was tailored to mimic the thermal wind relation $x \sim F$ for $F<1$ at vanishing asymmetry parameter $G$. For positive $G$ and larger values of $F$ not even the fixed point solutions of (1) reflect the thermal wind relation. The overall approximate validity of geostrophic balance, characterizing hydrodynamic 
(a)

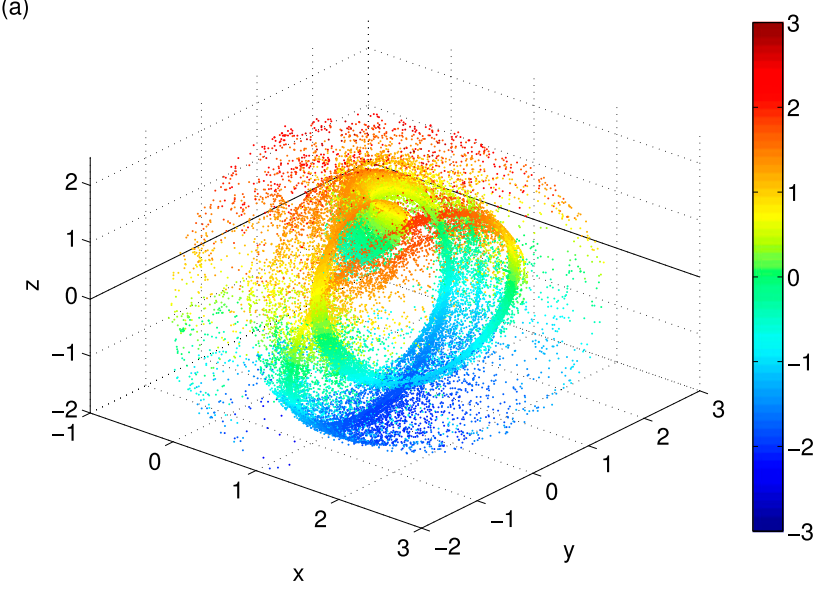

(b)

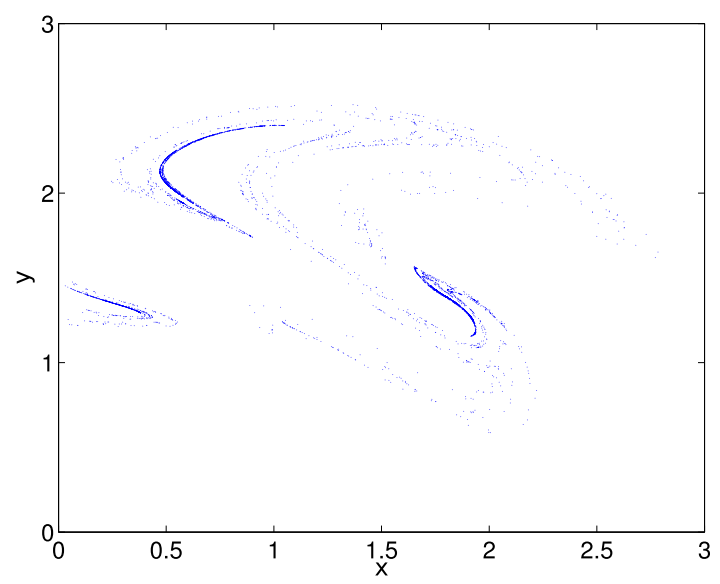

FIG. 1. Stroboscopic view at midwinter $(t \bmod T=T / 4)$ of the chaotic attractor of a stationary climate (a) in the full variable space (the $z$ component is color coded monotonically in the spectrum, reddish colors marking $z>2)$ and (b) on the $(x, y)$ plane. [The $z=0$ plane used to define the section of (b) is marked in (a)]. To generate both (a) and (b), a single trajectory of length of $10^{6} \mathrm{yr}$ is monitored and a transient segment of $5 \mathrm{yr}$ is cut out before plotting. For better visibility, we only included the first $10^{5} \mathrm{yr}$ in (a). The mean temperature contrast parameter is $F_{0}(t)=9.5$, independent of time.

models, cannot thus be expected to be present in this low-order model described by three ordinary differential equations.

To model seasonality, Lorenz uses a forcing (Lorenz 1990):

$$
F(t)=F_{0}(t)+A \sin (\omega t),
$$

with $A=2$ and a constant $F_{0}(t)$. The periodic form in (2) taken by him is centered on the fixed mean value $F_{0}(t)=7$ (Lorenz 1990) to describe the variation of the forcing over a year. We define $1 \mathrm{yr}$ to be $T=73$ time units, and thus $\omega=2 \pi / 73$ is set. Our calendar starts with year 0 , and this year begins at the time instant $t=$ 0 . Note that this time instant corresponds to an autumnal equinox, according to the expression in (2). Similarly, any time $t \bmod T=0$ coincides with autumnal equinoxes. Midwinters and midsummers then correspond to $t \bmod T=T / 4=18.25=0.25 \mathrm{yr}$ and $3 T / 4$ $=54.75=0.75 \mathrm{yr}$, respectively. As for the physical origin of the forcing we note that $F(t)$ may, among others, also contain the contribution of the varying $\mathrm{CO}_{2}$ content in association with the greenhouse effect. From this point on, $F(t)$ will be called the temperature contrast parameter. The model with (2) and $F_{0}(t)$ being constant is considered to represent a stationary climate.

To mimic a monotonous climate change, we set the mean value $F_{0}$ of the temperature contrast parameter to be time dependent. After the first $t_{\mathrm{st}}=100 \mathrm{yr}=7300$ time units of stationarity, when it takes the constant value of $9.5, F_{0}$ is chosen to be linearly decreasing:

$$
F_{0}(t)= \begin{cases}9.5, & \text { if } t \leq t_{\mathrm{st}} \\ 9.5-\frac{2}{t_{\mathrm{st}}}\left(t-t_{\mathrm{st}}\right), & \text { if } t>t_{\mathrm{st}} .\end{cases}
$$

This ramp form expresses that a climate change sets in at $t=t_{\mathrm{st}}=100 \mathrm{yr}$, and the temperature difference between the equator and the pole decreases; in a period of $150 \mathrm{yr}$ the mean value $F_{0}$ goes down to 6.5.

These equations were numerically solved by the classical fourth-order Runge-Kutta method with a fixed time step $d t=0.005 \approx 6.85 \times 10^{-5} \mathrm{yr}$.

\section{Characterization of a stationary climate}

The periodically driven model [governed by the dynamics (1)-(2) with $F_{0}(t)=$ const $]$ corresponds to a stationary climate. In such a three-variable system driven periodically in time with period $T=1 \mathrm{yr}$, there exists, at any fixed phase of the period, a unique (stroboscopic) attractor in the $(x, y, z)$ variable space that can be generated by a single long trajectory, after cutting out the initial transients. Figure 1a shows the $3 \mathrm{D}$ chaotic attractor at midwinter (i.e., $t \bmod T=T / 4$ ). The choice of taking a single "day" (more precisely, a particular phase) of the year is only a technical detail and is only aimed to give an impression not influenced by seasonality.

To obtain a planar view, a surface of section can also be taken on the plane $z=0$ with $\dot{z}>0$ (Lorenz 1984), and we restrict ourselves to this traditional choice. Points of the trajectory of Fig. 1a trace out in this section the pattern seen in Fig. 1b. Note that because of the periodicity of the forcing, the chaotic attractor is the 
(a)

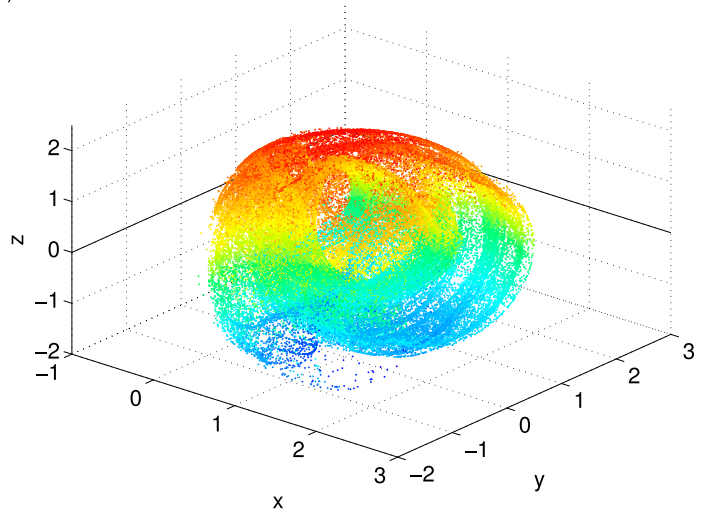

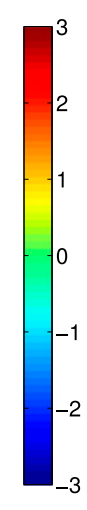

(b)

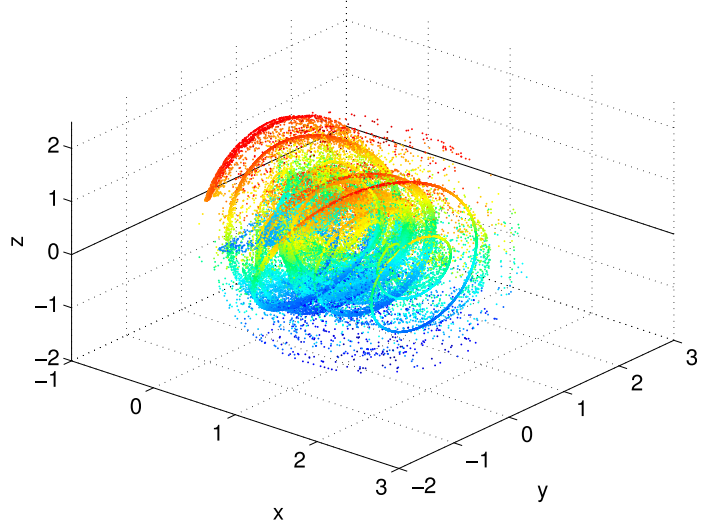

FIG. 2. The 3D snapshot attractor in the variable space. Numerically, it is traced out by an ensemble of $10^{6}$ realizations (see text) at the time instants (a) $t=250.25$ and (b) $t=250.75 \mathrm{yr}$, corresponding to midwinter and midsummer, respectively. The $z$ component is color coded monotonically in the spectrum, reddish colors marking $z>2$. (The $z=0$ plane used to define a slice of the snapshot attractor in Fig. 3 is marked in both panels.)

same when viewed after integer multiples of $T=1 \mathrm{yr}$, and the stroboscopic picture both in $3 \mathrm{D}$ and on the planar section does not change [see Ott (1993); for an illustration within our particular system, see also Bódai and Tél (2012)]. Had we taken a different day of the year for generating this set, the same would be true, just with a different pattern.

In Fig. 1b, we see clearly the characteristic filamentary, fractal pattern of chaotic attractors. The extension in both directions is larger than two units, which is an indication of the considerable internal variability of the dynamics. One particular feature is an "island" separated from the main body. It is located at about $x=0, y=1.4$, and can be considered as a sign of extremal behavior associated with very weak westerlies and below-average cyclonic activity. Most recently Ghil (2015) has given a definition of climate sensitivity by comparing probabilitybased internal variabilities of stationary climates.

Note that exactly the same attractor as in Fig. 1 can also be generated by an ensemble of trajectories started in the past and stopped at any time instant $t$ with $t \bmod T=$ $T / 4$, after an initial transient time interval.

\section{Characterization of a changing climate}

As discussed in the introduction, in the case of a perpetual shift of some of the parameters the snapshot picture is expected to provide a proper view. We illustrate different facets of this approach by applying the ramp in (3) in the temperature contrast parameter of the Lorenz model.

\section{a. The climatic snapshot attractor of the model}

A snapshot attractor is obtained numerically by tracking an ensemble of trajectories evolving under the set of (1)-(3). A uniform distribution of $N=10^{6}$ points is taken in a large box $[-1.5,3.5] \times[-2.5,2.5] \times$ $[-2.5,2.5]$ of the variable space at time $t_{0}=0.75 \mathrm{yr}$ (for technical convenience). The trajectories started with these initial conditions are followed up to a time $t$ that we choose to be longer than $t_{\text {st }}$ [after which the ramplike decrease (in what follows: climate change) sets in]. The endpoints of all the $10^{6}$ trajectories in the time instant $t$ trace out the snapshot attractor belonging to that time instant. Its form depends, of course, on the particular choice of $t$. The use of the term attractor is appropriate since the shapes are found to be independent of the set of initial conditions, as illustrated in the appendix. There is, of course, some time $t_{c}$ needed to come sufficiently close to the attractor. This convergence is expected, as in any dissipative system, to be exponentially fast. As we shall see in the next section, we estimate this time in the model to be $t_{c}=5 \mathrm{yr}$, which is much shorter than our choice for $t_{\mathrm{st}}$; therefore, for any $t>t_{\mathrm{st}}>t_{c}$ the points are expected to provide a faithful representation of the snapshot attractor in the climate change period.

As one of our typical examples, the snapshot attractor obtained this way for the last year of the investigated period (year 250, or year 150 after the onset of the climate change) is shown in the variable space in Fig. 2. The midwinter and midsummer instants belong to the left and the right panels, respectively. Both patterns are coil-like and are of the size of a few units in all three directions. The winter snapshot attractor appears, however, to be more extended. Besides illustrating how strong changes a snapshot attractor can undergo within a seasonal cycle, the figure shows that winter is much more active than summer, as also observed in the 
periodically driven Lorenz model (Lorenz 1990; Bódai and Tél 2012) (i.e., under a stationary climate).

It is to be emphasized that the scattered dots appearing in both panels (but more typical for the summer case) are not signs of slowly converging points, having not yet reached the attractor. As the appendix shows, taken at a different time instant (in year 150, i.e., in year 50 of the climate change period) for a better overview, scattered dots appear exactly in the same region even if different sets of initial conditions are chosen for the ensemble, and the convergence time remains to be about $5 \mathrm{yr}$. The scattered dots are thus signs of weakly occupied regions on both the midwinter and the midsummer snapshot attractors (and are consequences of the numerical use of a finite ensemble for the representation of the attractors). Section I of the supplemental material illustrates the drastic difference in the amount of information one can extract from the snapshot and from the traditional single-realization picture.

\section{b. Sliced snapshot attractor}

To gain a clearer view into the structure of the snapshot attractors, it is worth taking intersections of the 3D snapshot attractors with a surface. Planar slices taken with the conditions $z=0, \dot{z}>0$ are well defined and can numerically carry a sufficiently large number of points to be useful. We call the object obtained in this way the $(z=0)$ slice of the 3D snapshot attractor.

The tableau of Fig. 3 shows midwinter slices of the snapshot attractors in the first quadrant of the $(x, y)$ plane. Similarly as before, a particular day of the year is chosen in order to give an impression of the long-term temporal evolution of the snapshot attractor, not influenced by seasonality. This choice (i.e., that the system is viewed stroboscopically with respect to the periodic component of the forcing) is merely technical. This sequence of plots reflects fractal structures as clear as on the chaotic attractor of Fig. 1b, illustrating that such plots are the proper generalizations of this latter object. It also shows that on the same day of the years there are considerable changes in the variability over decades. A comparison of Figs. 1b and $3 \mathrm{a}$ leads to the conclusion that the island of special winter weather is still there after the onset of the climate change but its size becomes somewhat smaller, and the shrinking goes on such that a gap opens up eventually in the possible wind speeds. In Fig. $3 b x$ takes on values in the intervals $(0,0.4)$ and $(0.6,2.6)$ only. Parallel to this, the details of the filamentary structure of the bulk also change. The island is hardly visible in the next panel, and fully disappears by the 100th year after the onset of the climate change. No $x$ values are then realized below 0.4. In the remaining $50 \mathrm{yr}$ (Figs. 3e,f) the leftmost branch of the attractor is moving toward the $y$ axis, making the forbidden region in the wind speed smaller. Besides a continuous structural deformation, the maximal extension of the attractor changes in $x$ and $y$ from 2.8 to 2.5 and from 2.5 to less than 2 , respectively, during the whole climate change period. The evolution of the shape of the snapshot attractor in Fig. 3 is an indication of the change of the internal variability in a changing climate.

\section{c. The natural distribution}

The scattered dots in Fig. 2 clearly indicate that certain regions of the 3D snapshot attractor are visited much less probably than others. More systematically, defining a fine grid over the snapshot attractor, one can determine the number of points of the ensemble falling into the different boxes. This occupation probability can be considered, in the limit of a very fine grid and large ensemble, to be the natural distribution of the snapshot attractor. This distribution itself over the whole coil of the 3D snapshot attractor changes with time.

Since it is hardly possible to visualize a probability distribution defined on a three-dimensional support, it is worth evaluating analogous occupation probabilities over a two-dimensional grid covering the planar slices of the snapshot attractors. We call these the natural distributions on the slices of the snapshot attractors.

Figure 4 exhibits a few examples of such distributions. Note that they are all rather irregular, orders of magnitude differences can be present in neighboring boxes, which is a strong indication for their being of fractal measures (Ott 1993). Because of the parameter shift, the natural distribution turns out to change year by year even when sampled on the same day of the years. The first two panels belong to the slices of Figs. 3a and $3 \mathrm{~b}$. The other two panels exhibit distributions corresponding to time instants being in between those of Figs. $3 \mathrm{c}$ and $3 \mathrm{~d}$. These instants are intentionally chosen to be separated by a single year in order to illustrate how dramatic differences can show up in the natural distribution of the snapshot attractor in such a short time. It is remarkable that the support (the geometry of the snapshot attractor) hardly changes within the same time. In addition, the strong temporal dependence of the 3D snapshot attractor and its distribution is also reflected by the fact that the number of points falling into a neighborhood of the $z=0$ surface conditioned with $\dot{z}>0$ (i.e., the slice) changes considerably over the period investigated, as the data in the caption of Fig. 4 indicate.

The existence of the natural distribution of snapshot attractors is a central observation of our paper. As the example of Fig. 4 illustrates, this distribution is changing with time (i.e., it gives a response to the forcing). Nevertheless, it is unique at any time instant. Numerically, the only condition for this is that the ensemble used for 
(a)

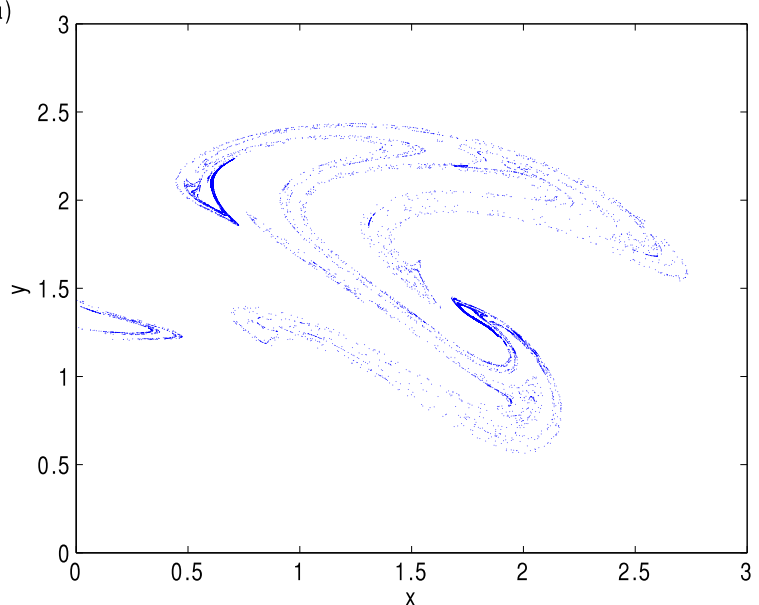

(c)

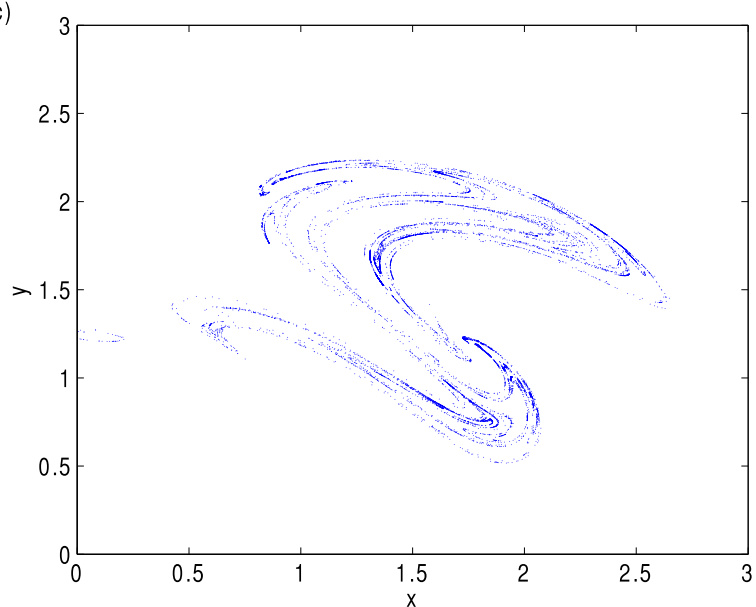

(e)

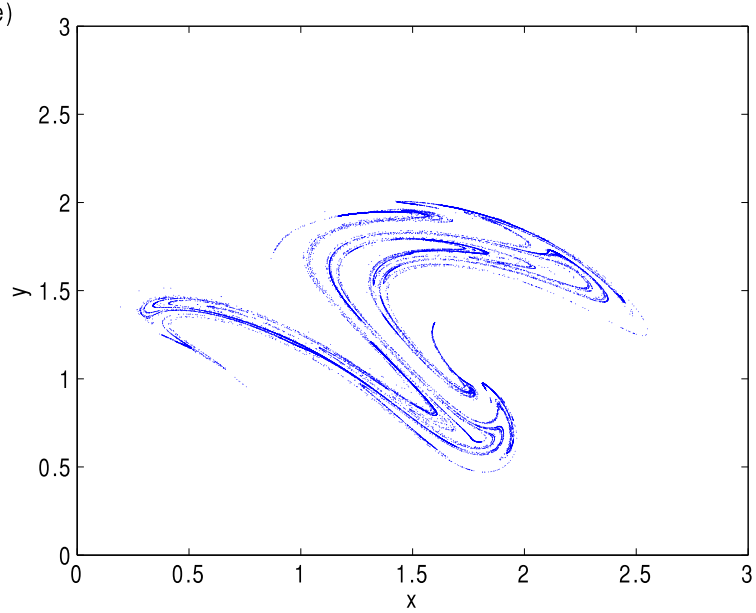

(b)

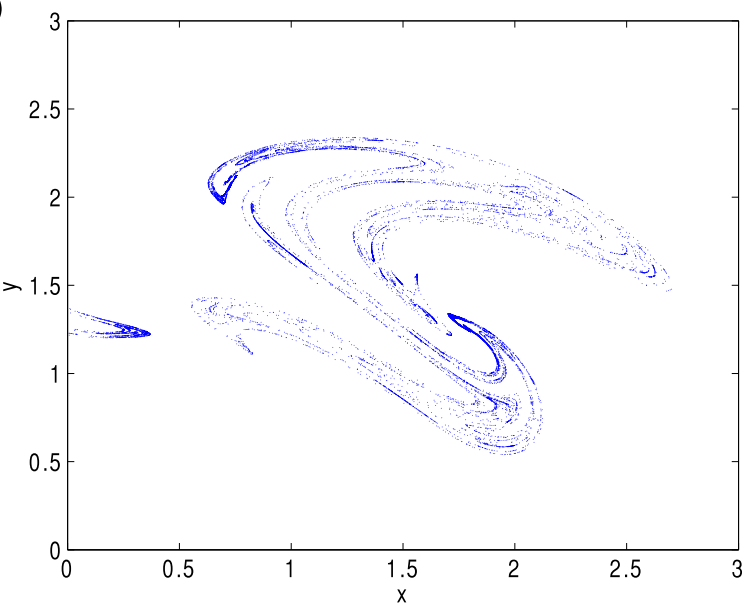

(d)

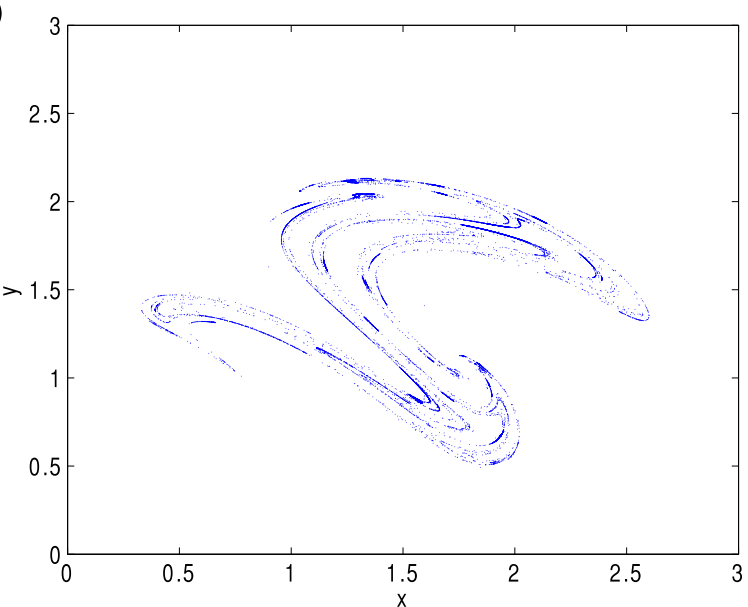

(f)

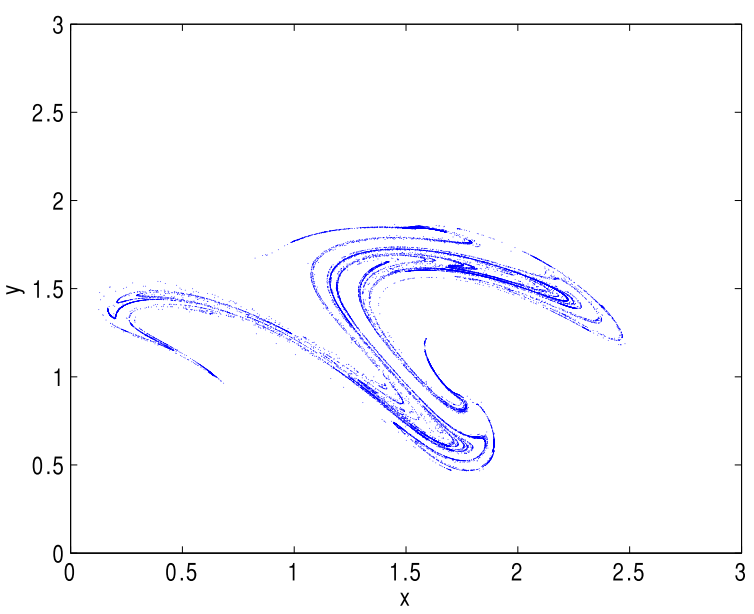

FIG. 3. (a)-(f) Midwinter slices of snapshot attractors in the plane of $z=0$ (conditioned by $\dot{z}>0$ ), generated with the ensemble of the realizations of Fig. 2. The first item of the tableau belongs to the time instant $t=125.25 \mathrm{yr}$ (midwinter of year 125 or of year 25 of the climate change period), the others, analogously, to the midwinter instants of years 50, 75, 100,125, and 150 of the climate change period. Panel (f) happens to be the slice of the 3D snapshot attractor shown in Fig. 2a. 
(a)

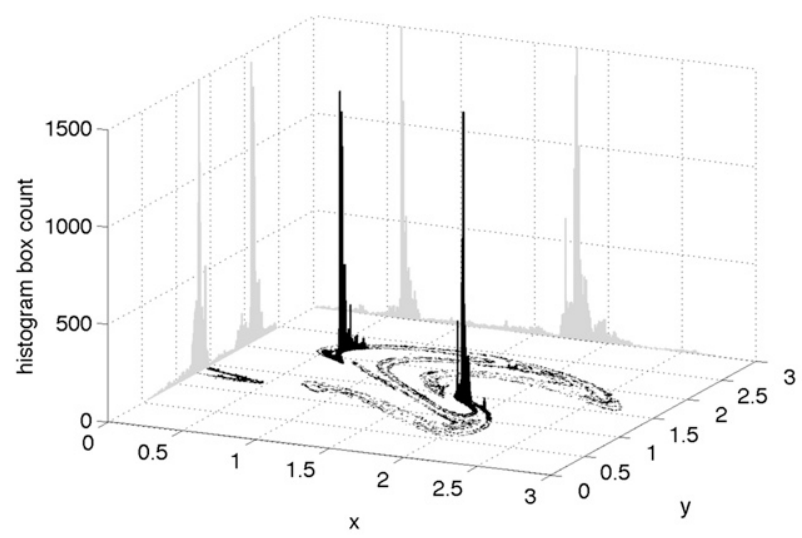

(c)

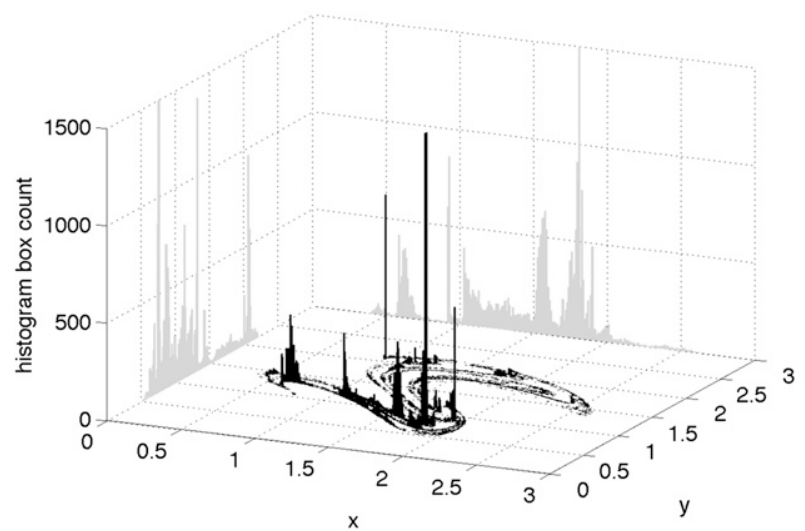

(b)

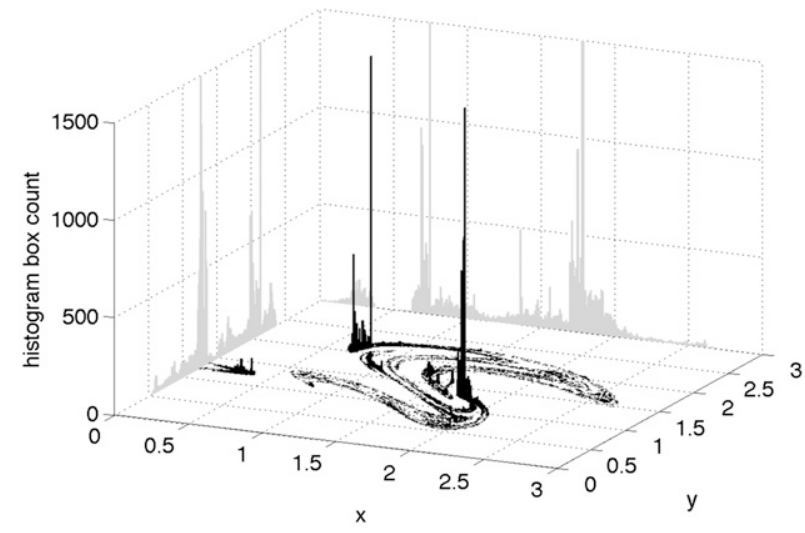

(d)

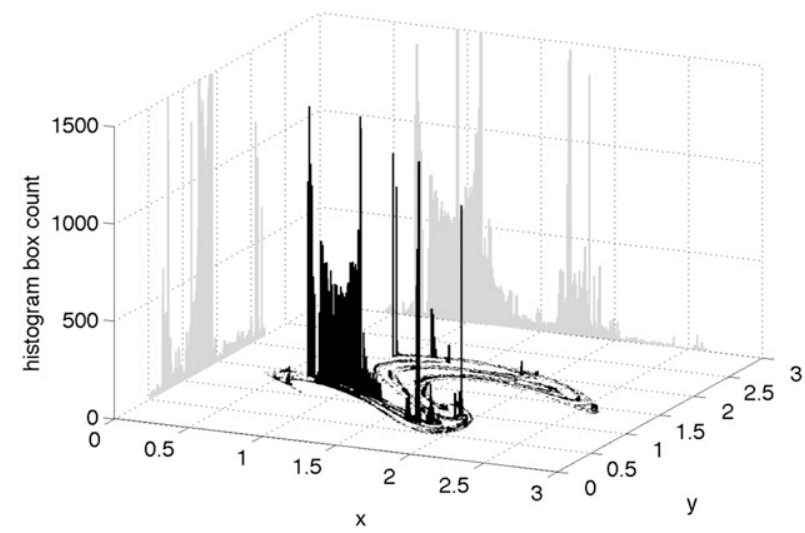

FIG. 4. Natural distribution on $z=0, \dot{z}>0$ slices of the snapshot attractor (generated with the ensemble of the realizations of Fig. 2) in the midwinter point of years (a) 25 , (b) 50 , (c) 88 , and (d) 89 of the climate change period. For better visibility, the occupation numbers are truncated at 1500 in the bins, whose linear size is chosen to be 0.01 . The number of points from which the distributions are generated in the slices in (a)-(d) is found to be 22 167, 25611,30142 , and 53 741, respectively. The distributions projected onto the $x$ and $y$ axes appear in gray in the back planes.

the generation of the probability distribution is initiated in the past farther back than a characteristic convergence time $t_{c}$, but otherwise both the time instant $t_{0}$ of initiation and the choice of the ensemble (the shape of the volume in the variable space in which the points are distributed, and the density of the points within this volume) is arbitrary, as illustrated in the appendix. There we also see that the convergence time $t_{c}$ is on the order of $5 \mathrm{yr}$, even if the ensemble is initiated after the onset of the climate change. The time $t_{c}$ can be interpreted as the time needed for a distribution to reach and become spread on the snapshot attractor. ${ }^{1}$ It is only this snapshot concept that

\footnotetext{
${ }^{1}$ The whole convergence is expected to be exponentially fast and $t_{c}$ is related in mathematical terms to the second discrete eigenvalue of the time evolution operator (Sell 1967a,b; Gaspard 2005).
}

makes possible a sound evaluation of statistical quantities, like averages and variances, and this can be done at any instant of time in a changing climate. We are not aware of any other tool that could be used for this purpose (i.e., for characterizing internal variability). \{Ensemble runs of short duration, i.e., ones lacking the convergence to the natural distribution of the snapshot attractor, can carry information that is very specific to the particular initial conditions, and also, of course, to the interval $\left[t_{0}, t\right]$ (where $\left.t-t_{0}<t_{c}\right)$. $\}$

\section{Ensemble and single-realization temporal statistics}

We now turn to the investigation of snapshotattractor-based statistics: averages and variances for the $x$ and $y$ variables will be evaluated with respect to the 
(a)

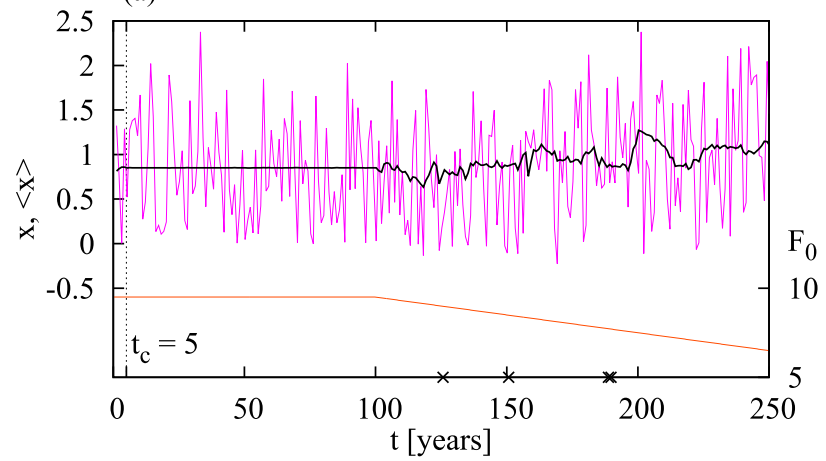

(b)

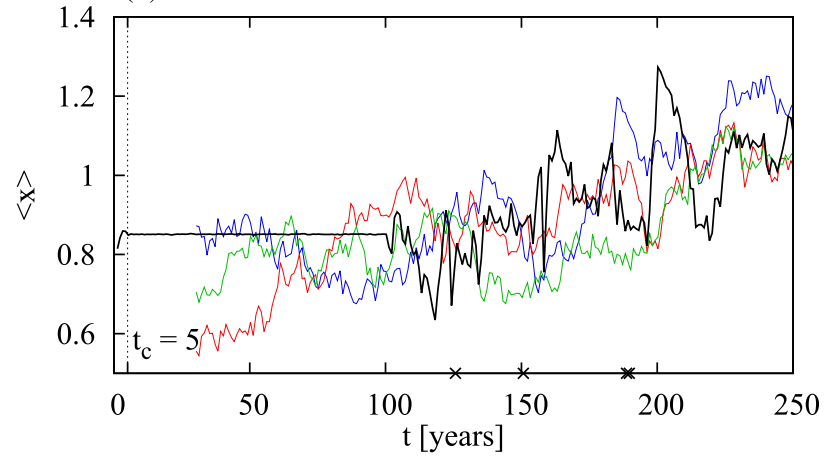

FIG. 5. The E (ensemble) average of the midwinter $x$ (black line) as a function of time and further characteristics. (a) A single-realization time series is marked by magenta, and (b) three different 30-yr single-realization temporal (SRT) averages (see text) are marked by blue, red, and green lines. The function $F_{0}(t)$ is also indicated in (a) in orange color, with the scale along the right edge. The convergence time $t_{c}$, after which averages do not change in the stationary period, can be seen at the beginning of the black line and is estimated to be $5 \mathrm{yr}$. The marked time instants (by $\times$ marks) along the $t$ axis correspond to the time instants considered in Fig. 4. (Years 88 and 89 are not clearly resolved.) The numerical ensemble, initiated at $t=0.75 \mathrm{yr}$ in the box $[-1.5,3.5] \times[-2.5,2.5] \times[-2.5,2.5]$, consists of $10^{6}$ individual realizations, one of which is plotted in every midwinter time instant in (a), and three of which are used for generating the SRT 30-yr averages in (b).

natural distribution of the snapshot attractor in the full $3 \mathrm{D}$ variable space. In addition, we compare the snapshot picture with averages and variances calculated along single realizations. More precisely, we numerically determine the statistics in a given time instant $t$ with respect to the natural distribution of the snapshot attractor, referred to as the ensemble statistics (E statistics), and the statistics taken along a single realization over the $30-\mathrm{yr}$ time interval in the past, called the single-realization temporal statistics (SRT statistics). (For such a set of points in a 151-yr interval, see Fig. SM1 of the supplemental material.) To suppress the effect of seasonality, we select, as earlier, one time instant $t \bmod T=$ const from each year.

In Fig. 5 we present numerically obtained averages. The black line corresponds to the $\mathrm{E}$ average of the variable $x$ at midwinter. As a contrasting reference, Fig. 5a also contains a single member of the ensemble, plotted in magenta color.

Observe that the black graph has two regions of different character. There is a plateau spanning the period of stationary climate illustrating the time independence of the E average. (The little fluctuations, though hardly if seen, originate in the finite size of the ensemble and represent the numerical uncertainty of the result.) At the onset of the climate change $\left(t=t_{\mathrm{st}}=100 \mathrm{yr}\right)$, a pronounced time dependence sets in, which is a forced response of the system. In the single realization (magenta line) of Fig. 5a, however, the fluctuations are so strong that hardly any change can be observed when entering the climate change period. This is the manifestation of the large internal variability on the snapshot attractor that hides the changes of the $\mathrm{E}$ average occurring in the climate change period from observers investigating the instantaneous values of only one realization. In other words, one cannot decide from a single realization alone when the temperature contrast parameter $F_{0}(t)$ begins to change, and how this change affects any statistical behavior.

The black E average evolves irregularly in the climate change period, exhibiting a slight overall tendency of increase. This increasing tendency is a sign of the lack of an overall thermal wind relation, a property of the model (1) mentioned in section 2. We emphasize that the irregularity is a consequence of the strong rearrangement of the probability on the snapshot attractor in time (cf. Figs. 4c,d). It should be noted that what we see is the average values taken with respect to the well-converged natural distribution on the snapshot attractor, examples of which (represented by their $z=0, \dot{z}>0$ slices, at least), belonging to the marked time instants along the $t$ axis, are exhibited in Fig. 4. In other words, what we see is a meaningful signal, even though it evolves irregularly in time.

SRT averages, with which we will now compare the $\mathrm{E}$ average, are similarly irregular, but are not meaningful from the point of view of probabilities. In Fig. 5b, the green line represents the 30-yr SRT average of the magenta single-realization line of Fig. 5a (plotted yearly at the endpoints of the 30 -yr intervals). ${ }^{2}$ The red and the blue lines are also 30-yr SRT averages, taken along realizations obtained from two other initial conditions, different from each other as well. These three realizations are, along with their 99997 companions,

\footnotetext{
${ }^{2}$ One can, of course, associate the average value to any point of the interval, among which the midpoint would be, perhaps, the most natural choice. The endpoint is, however, the traditional one.
} 
(a) Midwinter time instant

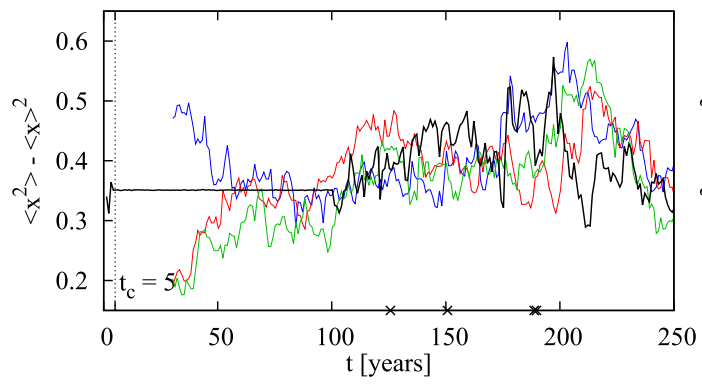

(b) Midsummer time instant

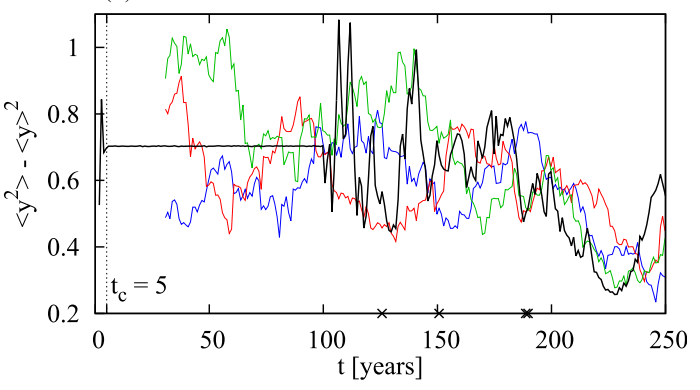

FIG. 6. The E variances (black lines) and three different 30-yr SRT variances (blue, red, and green lines) as a function of time. (a) Midwinter $x$ variances and (b) midsummer $y$ variances. The convergence time $t_{c}$ is also indicated. The marked time instants along the $t$ axis correspond to the time instants considered in Fig. 4 . The numerical ensemble is as in Fig. 5.

individual members of the ensemble. Note that any of the colored lines, corresponding to the 30-yr SRT averages of these realizations, can be considered as a "historically registered" climate (Stocker et al. 2013). The black E average line of Fig. 5a is reproduced in Fig. 5b on a different scale. We can observe that the SRT averages do not follow any fine structures in the $\mathrm{E}$ average, and even in the coarse structure substantial alterations occur. This is partially due to the large amount of information contained in 30-yr SRT averages that is obsolete from the point of view of the characterization of the present probabilities. What is more, they behave very differently in comparison with each other as well, indicating the lack of the representativity of one single realization for the ensemble behavior.

We note at this point that SRT averages sometimes produce strong signals suggesting changes that are false from the point of view of the snapshot picture. This is the most obvious in the stationary period of the climate, before year 100. For example, the red SRT average line in Fig. $5 \mathrm{~b}$ indicates an increase in the strength of the westerlies from 0.6 to 0.9 between years 50 and 90 . This might be interpreted as a climate change if one does not intend to think in terms of averages taken with respect to the natural probability distribution of the snapshot attractor. Unlike any SRT averages, the E average in Fig. 5 indicates properly the stationary nature of the dynamics and of the corresponding probability distribution.

In Fig. 6 we demonstrate similar features for variances, a specific measure of the internal variability of the dynamics. It is remarkable that the variances are by far not constant in the climate change period. In both panels the instantaneous E variances (black line) and the 30-yr SRT variances (blue, red, and green lines, corresponding in color to the realizations of Fig. 5) often go very far away from each other, and also these distances between different lines behave very irregularly (similarly as in Fig. 5).
The Lorenz system has been used here as a proof of concept to illustrate features of statistics taken with respect to the natural distribution of snapshot attractors, and the strong deviation of these statistics from 30-yr SRT statistics. We show that the situation does not change (see section II of the supplemental material) even if one takes time intervals of different length for SRT statistics.

\section{Final remarks}

In summary, we argued that the only appropriate probabilities, reflecting the internal variability of the dynamics, for an instant of time are described by the natural distribution of the snapshot attractor corresponding to this time instant. The temporal change of this distribution represents the forced response of the dynamics. Any ensemble-related probability distribution depending on the way of its generation (e.g., on the initial conditions) would be very specific to the particular procedure. The natural distribution of the snapshot attractor is, however, unique, as shown in the appendix. We have found that single-realization temporal statistics taken over 30 years are not capable of taking on numerical values that are reasonably close to those of the appropriate ensemble statistics derived from the natural distribution.

So far we have discussed only instantaneous values of any statistics. One is often interested, however, in the weather of a time interval (e.g., a season in the year, or a few decades). In such situations we first evaluate the quantity of interest ${ }^{3}$ on the time interval of interest along single realizations, and then calculate the statistics of this quantity over the ensemble of the realizations (representing the probabilities faithfully). We call such statistics

\footnotetext{
${ }^{3}$ This can be any arbitrary transformation of the trajectory values, including single-realization temporal (SRT) statistics.
} 
interval-wise-taken E (ensemble) statistics to avoid the use of the more technically sounding E statistics of SRT data that would be rather consistent with our terminology. The forced response of the dynamics is also naturally reflected in the time dependence of interval-wise-taken $\mathrm{E}$ statistics. We emphasize that the observation of this time dependence is conceptually different from observing the temporal change in some SRT statistics [by which a climate change is defined in Stocker et al. (2013)]. We show a few examples for interval-wise-taken E statistics and for their SRT counterparts in section III of the supplemental material, indicating that the deviations are practically as strong as for instantaneous statistics.

A natural question concerning the relevance of snapshot attractors and of their distributions is the robustness against noisy perturbations. Experience shows in any dynamical system that as long as the noise is weak, only the small-scale structure changes: fractality is washed out to a space-filling pattern but only below a short length scale in the variable space. This implies that averages can change only slightly in the presence of weak noise. A detailed investigation of noise effects from the point of view of snapshot attractors is given by Bódai et al. (2011b).

Another practical issue is the size of the ensemble needed to see the basic difference between singlerealization and snapshot-type results. We checked that the results for E averages obtained with $N=100$ members follow the "exact" $E$ averages (i.e., those that are obtained with $10^{6}$ members, e.g., the black line of Fig. 5), rather closely, deviating from it by about $10 \%$ of the $\mathrm{E}$ standard deviation. We thus conclude that the utilization of the snapshot approach is feasible even with moderate ensemble sizes. (Note that the use of large ensemble sizes appears to be hopeless in realistic climate simulations.) We are currently running an intermediate-complexity climate model in the spirit of the present paper, and our preliminary results are promising: even with a 10-member ensemble the numerical fluctuations around the ensemble average are found to be reasonably small. This ensemble average strongly differs from the values provided by the different realizations, and the time evolution of the 30 -yr temporal averages along individual realizations is again rather different both from that of the ensemble average and from those of each other.

An application of similar concepts appeared in a recent work (Ragone et al. 2014, manuscript submitted to Climate Dyn.) where predictions with an intermediatecomplexity climate model were investigated. For the application of the linear response theory, the authors needed the existence of a well-defined distribution in different time instants. They practically used the concept of the natural distribution of snapshot attractors, although only for the evaluation of expectation values, and interpreting standard deviations only as errors around them.

To incorporate more and more processes of the earth system, there is a natural need for the investigation of models with higher and higher complexities. A relevant question is the extent of the practical applicability of the snapshot framework to such models. In principle, all nonautonomous dynamical systems have snapshot attractors with a natural distribution on them. A case of particular interest is that when correlation times along trajectories are much longer in certain variables than in others (i.e., the case of time-scale separation). One might then wish to calculate the natural distribution restricted to the variables with short correlation times, keeping those with long correlation times approximately constant. This way one avoids the trajectories to visit those parts of the attractor that cannot be reached in some restricted time from a particular initial condition on the attractor (e.g., people are never interested in ice ages when thinking about the twenty-first century). To this end we propose to follow an ensemble of trajectories such that their initial conditions are distributed widely in the fast variables and are chosen according to given values in the slow variables. Although we propose to let also the slow variables evolve, they can be expected to remain approximately constant in the time interval of interest, which implies that the spread between different members of the ensemble remains small in these variables. Such an ensemble may be expected to converge to a "conditional" snapshot attractor in the fast variables after a certain convergence time. This construction might provide well-defined conditional probability distributions in situations where the correlation times in different variables are well separated. As for more general cases, we might hope that one can find in most of the models a reasonable cutoff in time for separating the degrees of freedom that are treated as frozen-in on the time scales of interest from those that are not.

Acknowledgments. Valuable comments by D. Berényi, U. Feudel, C. Franzke, M. Ghil, G. Halász, T. Haszpra, M. Herein, I. M. Jánosi, F. Lunkeit, J. Márfy, Z. Rácz, and M. Vincze are gratefully acknowledged. T. B. is grateful for support from the NAMASTE project led by Valerio Lucarini (under the ERC Grant 257106), and T. T. for support from the Alexander von Humboldt Foundation. This work was partially supported by the European Union and the European Social Fund through project FuturICT.hu (Grant TÁMOP-4.2.2.C-11/1/KONV-20120013). This work was also supported by the Hungarian Science Foundation under Grant NK100296. 
(a)

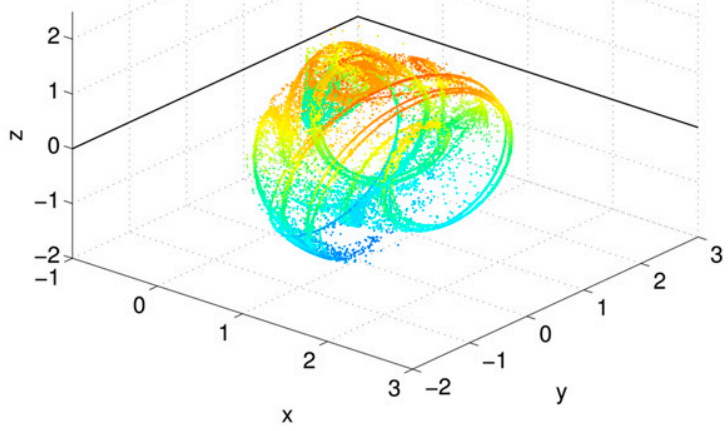

(c)

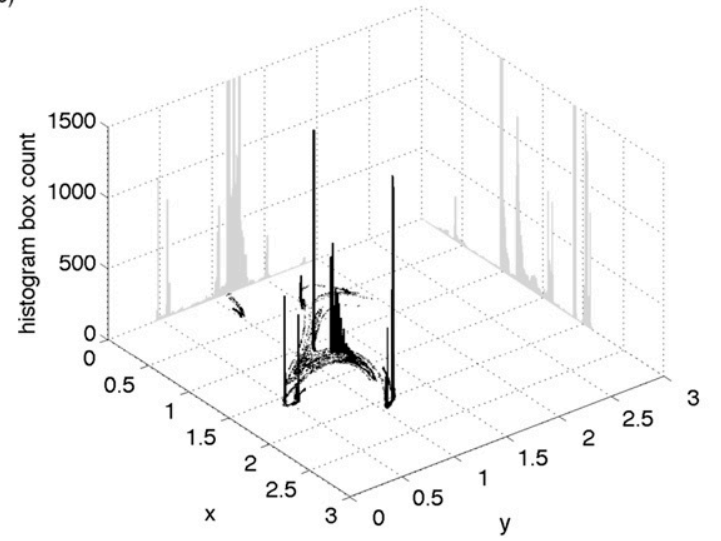

(b)

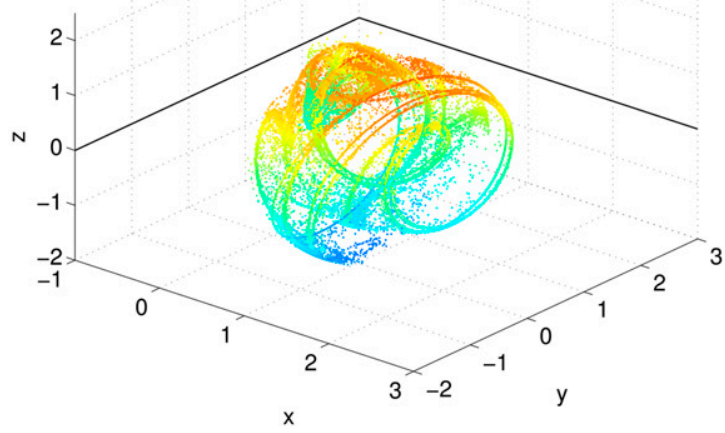

(d)

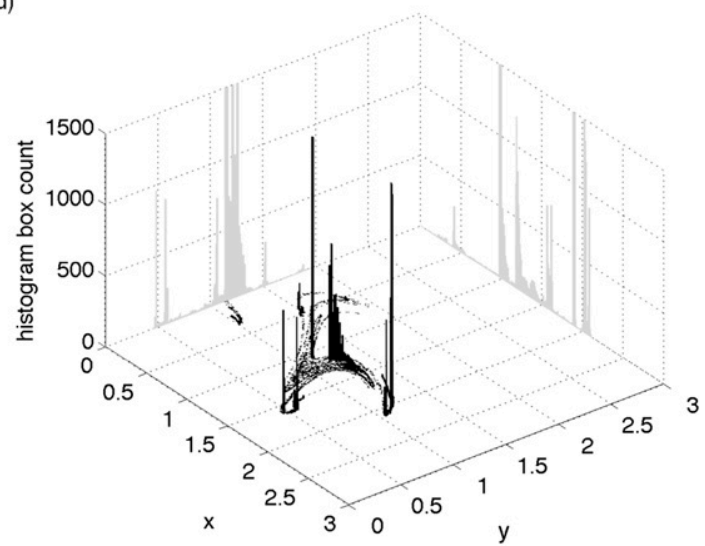

FIG. A1. Initial condition-independence I. The set of trajectory endpoints in the full variable space of two ensembles (of $10^{6}$ points each) at the midsummer time instant $t$ of year 150 , initiated in the box (a) $[-1.5,3.5] \times[-2.5,0] \times[-2.5,2.5]$ at time $t_{0}=110.75 \mathrm{yr}$ and (b) $[-1.5,3.5] \times[0,2.5] \times[-2.5,2.5]$ at time $t_{0}=130.75 \mathrm{yr}$. The $z$ component is color coded monotonically in the spectrum. (c),(d) The numerically determined distribution in bins of linear size 0.01 on the $z=0, \dot{z}>0$ slices of the ensembles of (a) and (b), respectively. For better visibility, the occupation numbers are truncated at 1500. The distributions projected onto the $x$ and $y$ axes appear in gray in the back planes. [The $z=0$ plane used to define a slice of the snapshot attractor is marked in (a) and (b).] Note that not even the times of initiation are identical. However, as both time evolutions are longer than $t_{c}$, the distributions are found to be identical.

\section{APPENDIX}

\section{Snapshot Attractors and Their Distributions Are Independent of Initial Conditions}

A basic property of any dissipative system is that the long-term dynamics is independent of initial conditions. This is why the underlying object can be called an attractor: it attracts all trajectories within a large region of the variable space, the basin of attraction. In spite of the nonautonomous character, this holds also for snapshot attractors (Ghil et al. 2008; Chekroun et al. 2011). Here we illustrate the attracting property for our model. To this end, we take two disjoint large boxes at different initialization times $t_{0}$ in which many initial conditions are distributed uniformly, and monitor both ensembles up to a given time instant $t$ in the set of (1)-(3).
(Our numerics do not indicate any simultaneous coexistence of different snapshot attractors.) We show results for the midsummer of year 150 (year 50 after the onset of the climate change) in Fig. A1: Figs. A1a,b present the position of the members of the two ensembles in 3D. Indeed, hardly any difference can be observed.

A perhaps even more relevant (but of course related) property is that the natural distribution (see section 4c) should also be independent of the initial conditions. For a clear visualization, as in sections $4 \mathrm{~b}$ and $4 \mathrm{c}$, we take the $z=0, \dot{z}>0$ slices of the attractors. The occupation numbers in grid cells over the $(x, y)$ plane are plotted for the two ensembles in Figs. A1c,d. Again, the agreement is striking. It is because of this agreement that we are allowed to speak about the natural distribution of the snapshot attractor. 


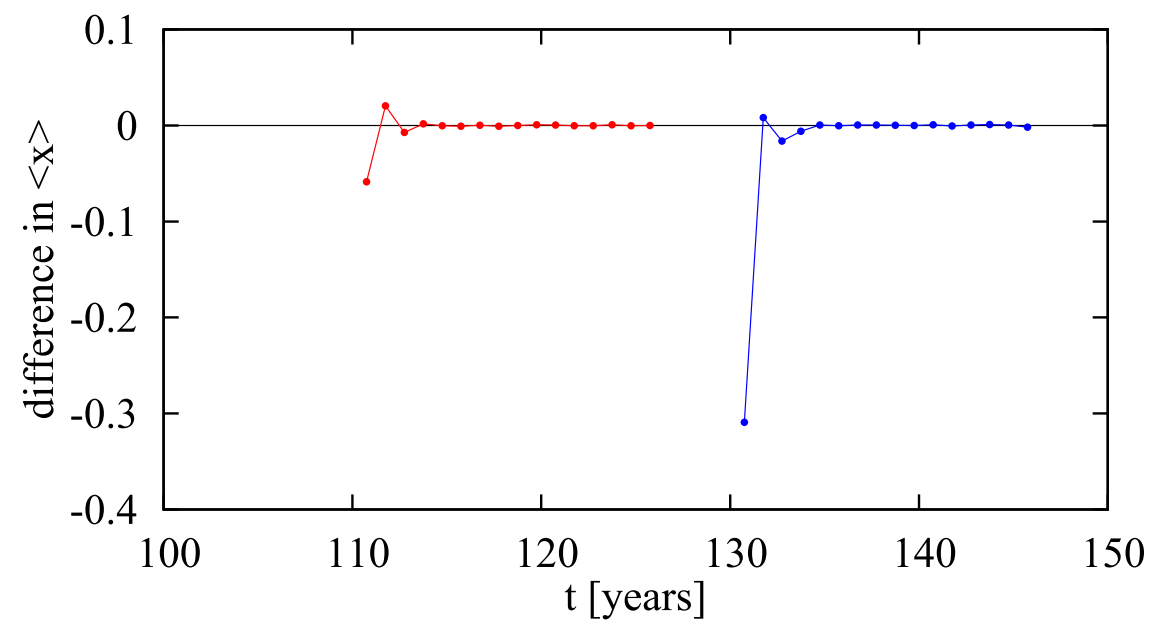

FIG. A2. Initial condition-independence II. The difference of the ensemble averages in the variable $x$ from that of the midsummer counterpart of Fig. 5, as a function of time, plotted at midsummer time instants. The red and the blue dots correspond to the ensembles of Figs. A1a and Figs. A1b, respectively, plotted in the first $15 \mathrm{yr}$ after initialization. The convergence time $t_{c}$ proves to be about $5 \mathrm{yr}$ for these ensembles, initiated in the climate change period.

This observation is further reinforced by Fig. A2 in which the difference of two ensemble averages ( $\mathrm{E}$ averages in the terminology of section 5 , the ones taken with respect to the ensembles of Fig. A1) from that of Fig. 5 are plotted as a function of time. The plot shows clearly that the average in $x$ of both ensembles of Fig. A1 converges within $t_{c}=5 \mathrm{yr}$ to the average of the ensemble initiated earlier in time. After the convergence period, the new ensemble averages stay together with the old one. These results provide a numerical support for the schematic diagram of Ghil and coworkers in Ghil et al. (2008) visualizing the convergence toward the snapshot attractors.

The findings illustrated by Figs. A1 and A2 illuminate the distinguishing relevance of the snapshot concept. The geometry and the natural distribution of snapshot attractors are free of any subjective choice (e.g., the region over which and the distribution according to which the initial conditions are taken). There exists thus (for $\left.t-t_{0}>t_{c}\right)$ an objective, a priori existing distribution belonging to any given time instant over which any statistics of interest can be evaluated.

\section{REFERENCES}

Arnold, L., 1998: Random Dynamical Systems. Springer-Verlag, $586 \mathrm{pp}$.

Bódai, T., and T. Tél, 2012: Annual variability in a conceptual climate model: Snapshot attractors, hysteresis in extreme events, and climate sensitivity. Chaos, 22, 023110, doi:10.1063/ 1.3697984.

— - G. Károlyi, and T. Tél, 2011a: A chaotically driven model climate: Extreme events and snapshot attractors. Nonlinear Processes Geophys., 18, 573-580, doi:10.5194/npg-18-573-2011.
,-- , and $-2011 \mathrm{~b}$ : Fractal snapshot components in chaos induced by strong noise. Phys. Rev., 83E, 046201, doi:10.1103/ PhysRevE.83.046201.

$\longrightarrow, \ldots$, and $—, 2013$ : Driving a conceptual model climate by different processes: Snapshot attractors and extreme events. Phys. Rev., 87E, 022822, doi:10.1103/PhysRevE.87.022822.

Carvalho, A. N., J. A. Langa, and J. C. Robinson, 2014: Attractors for Infinite-Dimensional Nonautonomous Dynamical Systems. Applied Mathematical Sciences Series, Vol. 182, Springer, 412 pp.

Chekroun, M. D., E. Simonnet, and M. Ghil, 2011: Stochastic climate dynamics: Random attractors and time-dependent invariant measures. Physica D, 240, 1685-1700, doi:10.1016/ j.physd.2011.06.005.

Crauel, H., and F. Flandoli, 1994: Attractors for random dynamical systems. Prob. Theory Relat. Fields, 100,365-393, doi:10.1007/ BF01193705.

, A. Debussche, and F. Flandoli, 1997: Random attractors. J. Dyn. Differ. Equations, 9, 307-341, doi:10.1007/ BF02219225.

Crucifix, M., 2013: Why could ice ages be unpredictable? Climate Past, 9, 2253-2267, doi:10.5194/cp-9-2253-2013.

Deser, C., R. Knutti, S. Solomon, and A. S. Phillips, 2012a: Communication of the role of natural variability in future North American climate. Nat. Climate Change, 2, 775-779, doi:10.1038/ nclimate1562.

—-, A. Phillips, V. Bourdette, and H. Teng, 2012b: Uncertainty in climate change projections: The role of internal variability. Climate Dyn., 38, 527-546, doi:10.1007/ s00382-010-0977-x.

Franzke, C. L. E., 2014: Warming trends: Nonlinear climate change. Nat. Climate Change, 4, 423-424, doi:10.1038/ nclimate2245.

Freire, J. G., C. Bonatto, C. C. DaCamara, and J. A. C. Gallas, 2008: Multistability, phase diagrams, and intransitivity in the Lorenz-84 low-order atmospheric circulation model. Chaos, 18, 033121, doi:10.1063/1.2953589.

Gaspard, P., 2005: Chaos, Scattering and Statistical Mechanics. Cambridge University Press, 496 pp. 
Ghil, M., 2012: Alfred Wegener medal lecture. EGU2012 Meeting, Vienna, Austria, EGU. [Available online at https://www.youtube. com/watch? $=$ czrvCdAqPbY.]

_ 2015: A mathematical theory of climate sensitivity or, how to deal with both anthropogenic forcing and natural variability? Climate Change: Multidecadal and Beyond, C. P. Chang et al., Eds., World Scientific Publishing Co./Imperial College Press, in press.

—- and R. Vautard, 1991: Interdecadal oscillations and the warming trend in global temperature time series. Nature, $\mathbf{3 5 0}$, 324-327, doi:10.1038/350324a0.

— M. D. Chekroun, and E. Simonnet, 2008: Climate dynamics and fluid mechanics: Natural variability and related uncertainties. Physica D, 237, 2111-2126, doi:10.1016/ j.physd.2008.03.036.

Hansen, J. L., and T. Bohr, 1998: Fractal tracer distributions in turbulent field theories. Physica D, 118, 40-48, doi:10.1016/ S0167-2789(98)00003-7.

Hasselmann, K., 1976: Stochastic climate models. Part 1. Theory. Tellus, 28A, 473-485, doi:10.1111/j.2153-3490.1976.tb00696.x.

Hawkins, E., and R. Sutton, 2009: The potential to narrow uncertainty in regional climate predictions. Bull. Amer. Meteor. Soc., 90, 1095-1107, doi:10.1175/2009BAMS2607.1.

Holton, J. R., 2004: An Introduction to Dynamic Meteorology. 4th ed. Academic Press, 535 pp.

Jacobs, J., E. Ott, T. Antonsen, and J. Yorke, 1997: Modeling fractal entrainment sets of tracers advected by chaotic temporally irregular fluid flows using random maps. Physica $D$, 110, 1-17, doi:10.1016/S0167-2789(97)00122-X.

Ji, F., Z. Wu, J. Huang, and E. P. Chassignet, 2014: Evolution of land surface air temperature trend. Nat. Climate Change, $\mathbf{4}$, 462-466, doi:10.1038/nclimate2223.

Károlyi, G., T. Tél, A. P. S. de Moura, and C. Grebogi, 2004: Reactive particles in random flows. Phys. Rev. Lett., 92, 174101, doi:10.1103/PhysRevLett.92.174101.

Kloeden, P., and M. Rasmussen, 2011: Nonautonomous Dynamical Systems. Mathematical Surveys and Monogr., No. 176, American Mathematical Society, 264 pp.

Ledrappier, F., and L.-S. Young, 1988: Dimension formula for random transformations. Commun. Math. Phys., 117, 529-548, doi:10.1007/BF01218383.

Leonardo, A., 1995: Numerical studies on the Lorenz-84 atmosphere model. M.S. thesis, Dept. of Mathematics, Utrecht University, and Koninklijk Nederlands Meteorologisch Instituut, $66 \mathrm{pp}$

Lorenz, E. N., 1984: Irregularity: A fundamental property of the atmosphere. Tellus, 36A, 98-110, doi:10.1111/ j.1600-0870.1984.tb00230.x.

_- 1990: Can chaos and intransitivity lead to interannual variability? Tellus, 42A, 378-389, doi:10.1034/ j.1600-0870.1990.t01-2-00005.x.

Masoller, C., and A. C. S. Schifino, 1992: Regular and chaotic behavior in the new Lorenz system. Phys. Lett. A, 167, 185-190, doi:10.1016/0375-9601(92)90226-C.

, - _ and L. Romanelli, 1995: Characterization of strange attractors of Lorenz model of general circulation of the atmosphere. Chaos, Solitons Fractals, 6, 357-366, doi:10.1016/ 0960-0779(95)80041-E.
Neufeld, Z., and T. Tél, 1998: Advection in chaotically timedependent open flows. Phys. Rev., 57E, 2832, doi:10.1103/ PhysRevE.57.2832.

Nicolis, C., S. Vannitsem, and J.-F. Royer, 1995: Short-range predictability of the atmosphere: Mechanisms for superexponential error growth. Quart. J. Roy. Meteor. Soc., 121, 705-722, doi:10.1002/qj.49712152312.

Ott, E., 1993: Chaos in Dynamical Systems. Cambridge University Press, 385 pp.

Paillard, D., 2008: From atmosphere, to climate, to earth system science. Interdiscip. Sci. Rev., 33, 25-35, doi:10.1179/ $030801808 X 259943$.

Pielke, R. A., and X. Zeng, 1994: Long-term variability of climate. J. Atmos. Sci., 51, 155-159, doi:10.1175/1520-0469(1994)051<0155 LTVOC $>2.0 . \mathrm{CO} ; 2$

Pierini, S., 2012: Stochastic tipping points in climate dynamics. Phys. Rev., 85E, 027101, doi:10.1103/PhysRevE.85.027101.

Provenzale, A., and N. J. Balmforth, 1999: Chaos and structures in geophysics and astrophysics (Woods Hole lecture notes). WHOI, $13 \mathrm{pp}$. [Available online at http://www.whoi.edu/fileserver.do? $\mathrm{id}=21476 \& \mathrm{pt}=10 \& \mathrm{p}=17353$.]

Reichl, L. E., 1998: A Modern Course in Statistical Physics. 2nd ed. Wiley, $822 \mathrm{pp}$.

Roebber, P. J., 1995: Climate variability in a low-order coupled atmosphere-ocean model. Tellus, 47A, 473-494, doi:10.1034/ j.1600-0870.1995.t01-3-00006.x.

Romeiras, F. J., C. Grebogi, and E. Ott, 1990: Multifractal properties of snapshot attractors of random maps. Phys. Rev., 41A, 784, doi:10.1103/PhysRevA.41.784.

Sell, G. R., 1967a: Nonautonomous differential equations and topological dynamics. I. The basic theory. Trans. Amer. Math. Soc., 127, 241-262.

_ 1967b: Nonautonomous differential equations and topological dynamics. II. Limiting equations. Trans. Amer. Math. Soc., 127, 263-283.

Shil'nikov, A., G. Nicolis, and C. Nicolis, 1995: Bifurcation and predictability analysis of a low-order atmospheric circulation model. Int. J. Bifurcation Chaos Appl. Sci. Eng., 05, 1701, doi:10.1142/ S0218127495001253.

Sommerer, J. C., and E. Ott, 1993: Particles floating on a moving fluid: A dynamically comprehensible physical fractal. Science, 259, 335-339, doi:10.1126/science.259.5093.335.

Stocker, T. F., and Coauthors, 2013: Climate Change 2013: The Physical Science Basis. Cambridge University Press, 1535 pp. [Available online at www.climatechange2013.org/images/report/ WG1AR5_ALL_FINAL.pdf.]

Taylor, K. E., R. J. Stouffer, and G. A. Meehl, 2012: An overview of CMIP5 and the experiment design. Bull. Amer. Meteor. Soc., 93, 485-498, doi:10.1175/BAMS-D-11-00094.1.

Tél, T., and M. Gruiz, 2006: Chaotic Dynamics: An Introduction Based on Classical Mechanics. Cambridge University Press, 393 pp, doi:10.1017/CBO9780511803277.

Young, L.-S., 2002: What are SRB measures, and which dynamical systems have them? J. Stat. Phys., 108, 733-754, doi:10.1023/ A:1019762724717.

Yu, L., E. Ott, and Q. Chen, 1990: Transition to chaos for random dynamical systems. Phys. Rev. Lett., 65, 2935-2938, doi:10.1103/ PhysRevLett.65.2935. 